\title{
A Survey of Contextual Handwritten Recognition Systems based HMMs for Cursive Arabic and Latin Script
}

\author{
Mouhcine Rabi \\ Laboratory IRF-SIC, faculty of \\ sciences, Ibn Zohr University \\ Agadir, Morocco
}

\author{
Mustapha Amrouch \\ Laboratory IRF-SIC, faculty of \\ sciences Ibn Zohr University, \\ Agadir, Morocco
}

\author{
Zouhir Mahani \\ High school of technology. \\ Ibn Zohr University, Agadir, \\ Morocco
}

\begin{abstract}
Offline handwriting recognition has become lately a very popular research area and the number of its possible application is very large. Most recognition system are based on modeling characters to recognize, then the concatenation of these models to recognize a word, while modeling character allows deformations related to its context. This paper provides a survey of handwritten recognition systems based on context-dependent character modeling to account possible deformations related to its context. It examines the literature on the most significant work in contextual handwritten text recognition for two different alphabets, Latin and Arabic. Finally discussing the comparative results to achieve a comprehensive summary of the various approaches and systems taking account the character's context which could help open up some interesting new prospects.
\end{abstract}

\section{Keywords}

Offline handwriting Recognition, Latin, Arabic, Context, Cursive

\section{INTRODUCTION}

Handwritten character recognition is a process of transforming handwritten text into machine executable format. There are mainly three steps in pattern recognition: observation, pattern segmentation and pattern classification. Character's recognition has become very interesting topic in pattern recognition for the researchers during last few decades. In general, handwritten recognition is classified into two types as on-line and off-line recognition methods [01]. Offline handwriting recognition involves the text's automatic conversion into an image into letter codes which are usable within computer and text-processing applications. The data obtained by this form is regarded as a static representation of handwriting. But, in the on-line system, the two dimensional coordinates of successive points are represented as a function of time and the order of strokes made by the writer are also available. Offline character recognition is comparatively more challenging due to shape of characters, great variation of character symbol, different handwriting style and document quality.

Several applications including mail sorting, bank processing, document reading and postal address recognition require offline handwriting recognition systems. As a result, the off-line handwriting recognition continues to be an active area of research towards exploring the newer techniques that would improve recognition accuracy.

The study defines the five major stages in any handwritten character recognition which is shown in Figure1.

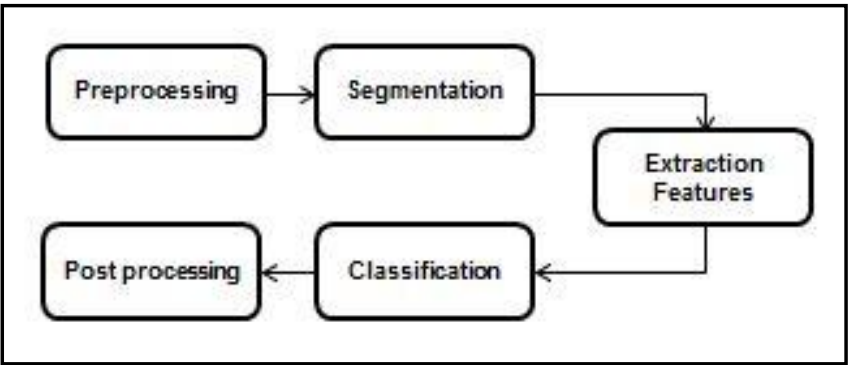

Figure 1 : stages of character recognition

In image preprocessing we apply a series of operations like Binarization, Complement, size normalisation, Morphological operation, noise removal using filters, thresholding, skeletonization, thinning, cleaning techniques and filtering mechanisms on scanned image which are taken as input. It makes the input image easier to process in order to increase the overall efficiency of recognition system [02].

Segmentation is process of extracting the basic constituent symbols of the script. Image is subdivided into many parts so that each part of the image is readable. To accomplish this task the image is subdivided considering three aspects, i.e. line wise segmentation, word wise segmentation and character wise segmentation.[03]

Feature extraction is a special form of dimension reduction. This approach is useful when image sizes are large and a reduced feature representation is required to quickly complete tasks such as image matching and retrieval [04][05].

The classification stage is the decision making stage of the recognition system, is the process of assigning the sensed data to their corresponding class with respect to groups with homogeneous characteristics, with the aim of discriminating multiple objects from each other within the image. There are many existing techniques available for handwriting classification.[06]

The last stage of the character recognition system is Postprocessing. It prints the corresponding recognized characters in the structured text form. The accuracy of character recognition stages can be improved if the semantic information is available up to great extent.

Researches have tried various approaches employing various techniques for pre-processing, features extraction and classification [07][08].

The cursive nature of handwriting text, the word's variability, letter shapes are context sensitive, inter and intra word spaces, the skew and slant of characters and words makes the construction of offline recognition system a challenging task, opening the way to a new approaches using 
environment context of characters to build a more precise and effective models.

The remainder of this paper is organized as follow. Section 2 presents a motivation of using contextual models for recognition. Section 3 the literature on the most significant work in contextual handwritten text recognition. The performance of the recognition systems and the comparative results are shown and analyzed in section 4 . The paper finally concludes with some conclusions and perspectives.

\section{CONTEXTUAL RECOGNITION: MOTIVATION}

Context is a significant element in the recognition process.

When people read handwriting, they look at entire words or even the entire document to correctly identify what is written. They can read a sentence and understand illegible words within the context of the document

In the same way, recognition engines use context as an effective and flexible tool to compensate for the ambiguity of handwriting and to improve recognition accuracy.

The principle of context-dependent modeling of characters is quite natural, as it is obvious that our way of writing a character within a word evolves with its adjacent letters. An example of contexts influencing letters's shape can be seen on figure 2 for the lowercase character "s". Considering that the two words have been written by the same person, we can easily imagine that the letters's shape is highly variable in a large database. Moreover a character in Arabic script can take different forms based on its position in the word. Parts of these character shapes are similar for the different forms of the same character and also between the different characters and their contextual forms.

The idea of context-dependent modeling is that, instead of defining a word as a succession of characters, we define a word as a succession of characters and their contexts.

Indeed, in cursive scripts, the character's shape is dependent of its context, usually described by the character preceding and following it. This is illustrated is figure 2 .

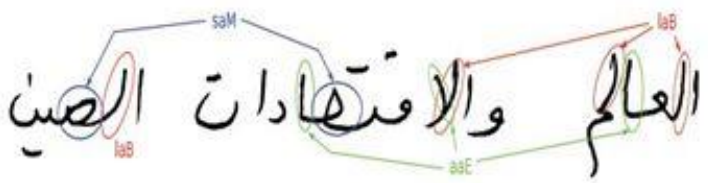

(a)

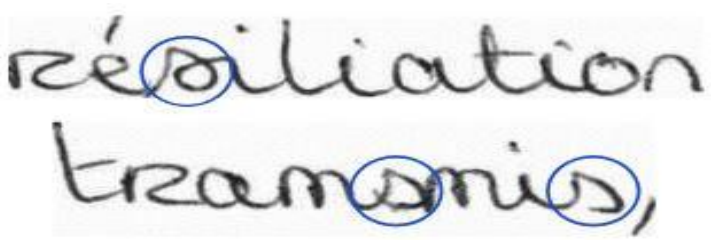

(b)

Figure 2: (a) Arabic words 'الصين' 'الاقتصادات' , and 'العالم'

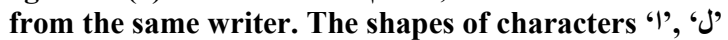
and ' $ص$ ' change dependent context

(b) French words 'résiliation' and 'transmis' from the same writer. The shape of the character ' $s$ ' is context dependent.[09]
The character's modeling allows deformations related to its context. To account possible deformations, contextual modeling of characters is opted. Taking account the environment of a character for her modeling allows building more accurate and precise models to achieve an effective handwritten recognition system.

\section{HANDWRITTEN RECOGNITION SYSTEMS USING CONTEXT}

Various contextual approaches employing various techniques are proposed:

\subsection{Gernot A.Fink [10]}

Gernot A.Fink and Thomas Plötz tried to shed some light on the inherent difficulties faced when using context-dependent models for offline handwriting recognition, the system had evaluated using IAM database [11]

Baseline system: first, text lines are extracted from the input documents. The lines are normalized with respect to baseline orientation, slant, and estimated average character width. A pre-segmentation into words is, however, not attempted. From the normalized text lines feature vector sequences are extracted using the sliding-window approach. For every analysis window a set of geometric and their discrete derivatives is computed.

The HMMs for the 7485 words in the lexicon are constructed from 75 basic units representing context independent characters, numerals, punctuation symbols, and white space. The model for the word "Brazil" is, e.g., formed by concatenating the context independent character models:

$$
\square(\text { Brazil })=\square(\text { B })^{\circ} \square\left(\text { r) }{ }^{\circ} \square \text { (a) }{ }^{\circ} \square \text { (z) }{ }^{\circ} \square \text { (i) }{ }^{\circ} \square\right. \text { (l) }
$$

The models have Bakis-type topology. The number of states depends on the length of the associated segments in the initialization data resulting in 2248 states. The context independent HMMs as well as all context dependent models are semi-continuous sharing a set of $1.5 \mathrm{k}$ Gaussian densities with diagonal covariance matrices.

Context dependent sub-word units: All occurrences of characters with different left and right context found in the training data were established as context dependent models. The word "Brazil" is now built as:

$\square($ Brazil $)=\square(\# / \mathrm{B} / \mathrm{r}){ }^{\circ} \square(\mathrm{B} / \mathrm{r} / \mathrm{a}){ }^{\circ} \square(\mathrm{r} / \mathrm{a} / \mathrm{z})^{\circ} \square(\mathrm{a} / \mathrm{z} / \mathrm{i}){ }^{\circ}$ $\square(\mathrm{z} / \mathrm{i} / \mathrm{l})^{\circ} \square(\mathrm{i} / \mathrm{l} / \#)$

Where $\square(\mathrm{r} / \mathrm{a} / \mathrm{z})$ denotes a model for the base character a with left context $\mathrm{r}$ and right context $\mathrm{z}$ and \# represents the word boundary.

Clustering procedure: For this overall model an intermediate parameter set is estimated. Afterwards, all states corresponding to the same position in models with the same base character are subject to an agglomerative clustering.

To guide the clustering by reducing the degrees of freedom by grouping contexts on the symbolic level. As it is far from obvious how classes of similar context should be defined for offline data we came up with 5 rather simple context categories that - to some extent - show similar geometric properties: characters that occupy the core area only (core), characters with ascenders (asc), descenders (desc), or both (adc), numerals (num), and capitals (cap). Punctuation symbols and white space were treated separately. "Brazil" is now defined as: 


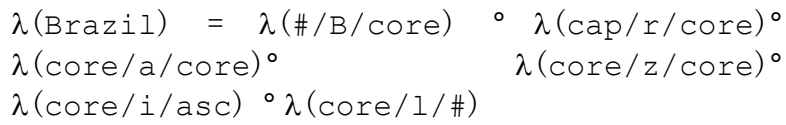

\subsection{Ramy .El-Hajj [12]}

R.El-Hajj, C.Mokbel and L.Likforman-Sulem proposed an enhanced version of reference system of Arabic handwriting recognition system using contextual character models.

An enhanced reference system which takes into account character overlap. It is based on the use of contextual character models. Characters which may include ascending or descending strokes in a near spatial context are represented by specific HMM character models. The set of character models is thus increased. It can be noted that these additional models differ from those of Arabic characters whose shapes are context sensitive, according to the position of the character in a word (beginning, middle, end): these various shapes are already taken into account in the reference system. The experiments are conducted on the benchmark IFN-ENIT database [13]

Reference System :The reference HMM system is based on an analytical strategy with implicit segmentation [14].

The system takes as input binary word images. The preprocessing phase consists of searching for the lower and the upper baselines.

The feature extraction stage consists of extracting a sequence of feature vectors by dividing the word image into vertical overlapping frames. In each window a set of 28 features are extracted. These features represent the local distributions of densities and the configurations of foreground pixels which capture the type of strokes (curved, oriented, vertical, and horizontal).

Contextual character modeling system : Character and word models: 167 character HMM models are constructed, the 29 basic shapes, the 94 context sensitive shapes (vary according position ; beginning, middle or end), 43 additional shapes which represent characters with additional diacritical marks such as shadda and 1 space model.

The topology of character models is left-right with four states (Figure 3 ). The probability densities of observations in each state are modeled as a mixture of three Gaussian distributions.

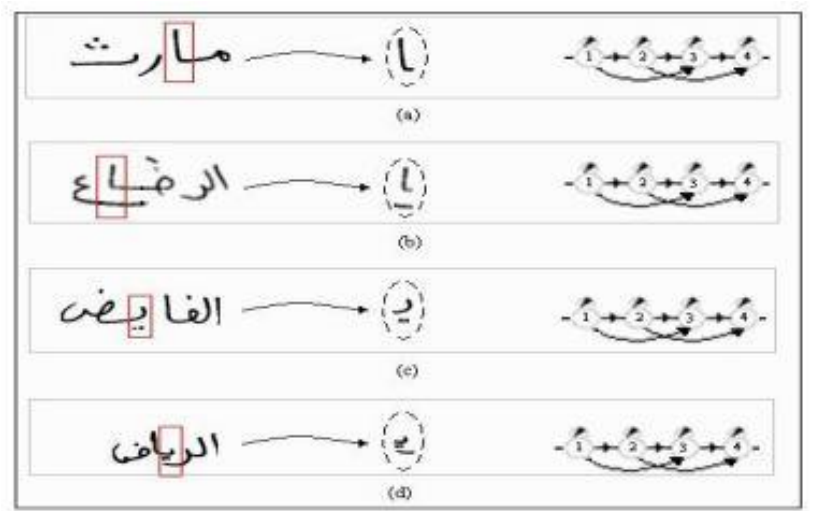

Figure3: Examples of character models. (a) and (c) are a non-contextual models. (b) and (d) are additional contextual models[12]

El-Hajj [12] simplify the tri-character approach and present a parallel alternative in which select manually the characters which are overlapped by neighboring strokes and construct the corresponding contextual model. This leads to a lower number of additional models than with the tri-character approach because characters are modeled by at most two models. The first model corresponds to a shape in the original set (which may be basic, context-sensitive or with diacritical mark). The second model corresponds to a shape overlapped by neighboring descenders. This leads to 44 additional models for the number of name classes considered.

\subsection{Anne-Laure Bianne [15]}

Anne-Laure Bianne presents an HMM-based recognizer for the offline recognition of hand written words. Word models are the concatenation of context-dependent character models: the trigraphs. Due to the large number of possible contextdependent models to compute, a clustering is applied on each state position, based on decision trees.

Context Independent handwriting recognition system: The first steps of a recognition system consist in preprocessing the input images and extracting features. The preprocessing step is limited to slant correction images since slant is observed in the Rimes database and the skew are negligible.

Feature extraction is based on the work of El-Hajj et al [12] A Sequence of feature vectors are extracted from left to right through overlapping windows. The window is a frame divided into a fixed number of cells. Within each window a set of 28 features is extracted. A number of features are baseline dependent. The grey-level images are binarized by the Otsu method in order to compute features related to foreground/background transitions and pixel configurations.

Context-independent system uses the analytical strategy and is segmentation- free. A word is modeled by the concatenation of its compound characters. All character models share the same HMM topology: 8 emitting states, left-right transitions with one skip allowed. The observation probability density for each state is a mixture of 20 Gaussian distributions. HMM models are trained using the BaumWelch algorithm and decoding is made with the Viterbi algorithm.

Since the baseline system is context-independent, a single model corresponds to each letter. In the following we call these context-independent models 'monographs'. A total of 66 (40) are defined different case sensitive (resp. case insensitive) monographs, which can be letters, numerals, or symbols (hyphen, apostrophe, etc.).

Context dependent handwriting recognition system: The idea of context-dependent modeling is that, instead of defining a word as a succession of characters, we define a word as a succession of characters and their contexts. The monographs are replaced by trigraphs. A left context is defined with a minus "-" sign, and a right context with a plus "+" sign. For example, on figure 4, the letter "i" surrounded by a "s" and an "e" is now written : s - i + e. Similarly, the letter " $\mathrm{d}$ " surrounded by a silence and an " $\mathrm{i}$ " is now written : $0-d+i$. 


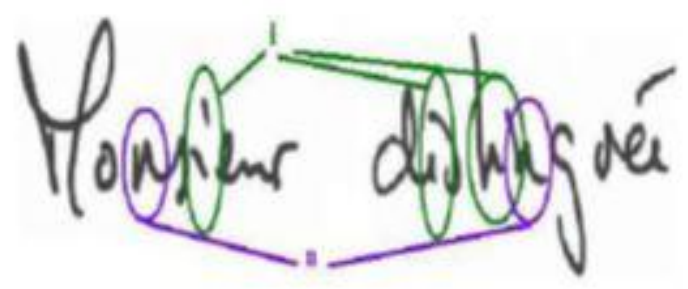

Figure 4: influence of context for handwriting on two words written by the same person ( "Monsieur", "distinguée")[15]

A drawback of building context-dependent models is that the number of HMM parameters related to all letter contexts becomes huge and there may be a lack of training data. This number of parameters can be reduced by sharing them between several models.

To build trigraph models and to share parameters between these trigraphs, two types of parameters are shared: transition matrices are tied, and state models are clustered with an original decision tree-based clustering method.

For this system, trigraphs are modeled instead of monographs. The first step of the training phase is the clustering of models to reduce the dimension of the problem. Once the processes of transition matrix and state tying are completed, the system is ready to finish the training of the models with a high level of refinement. Using the BaumWelch algorithm for model re-estimation, the number of Gaussian distributions per state is increased up to 20, topology of 8 emitting states with 20 Gaussian distributions per state for all the trigraphs.

\subsection{Olivier Morillot [16]}

This paper describes a global approach for the recognition of handwritten lines. Its main contribution is to extend the context-dependent HMMs word recognition system to the line level without segmentation into words. Thus, the authors describe a complete solution for mail recognition: original preprocessing, context-dependent HMMs and an optimized bigram language model.

The lines are modeled from the concatenation of words separated by spaces models (model of Silence). The word models are in turn obtained by the concatenation of character patterns the component.

Contextual models of character: A character is modeled according to its context, i.e the letters that surround it. The monographs are replaced by trigraphs, where the central character is added character left and right (left and right context).

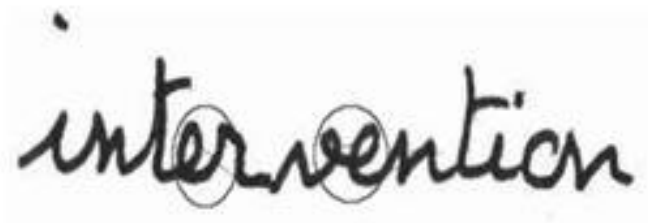

\section{Figure 5: Different shapes of character ' $e$ ' according its context[16]}

However, it is not possible to consider all the possible trigraphs, this would greatly increase the number of parameters to estimate. It would increase from 91 monographs (uppercase and lowercase letters, accented characters, numbers and special characters) $91^{3}$ trigraphs. Fortunately, not all trigraphs are observable. For example, analysis of a dictionary of 11000 words only shows 9400 different trigraphs. It is also possible to reduce the number of parameters to be estimated by grouping some trigraphs (Bianne-Bernard et al., 2011)[15].

Language model: The passage of the recognition of the level word to line level provides the ability to model the successive words with a language model. The objective of the system is to learn what are the most likely words estates to improve the recognition made by the optical model. So a bad word recognized based HMM can thus be corrected through context.

The estimate of the optimal sequence of words is given $\hat{\mathrm{W}}$ :

$\hat{\mathrm{W}}=\operatorname{argmax} P(\mathrm{~W} \mid \mathrm{X})=\operatorname{argmax} P_{\text {optic }}(\mathrm{X} \mid \mathrm{W}) P_{\text {grammar }}(\mathrm{W})$

Where the likelihood $P_{\text {optic }}(\mathrm{X} \mid \mathrm{W})$ of the observation sequence $\mathrm{X}$ for the sequence of words $\mathrm{W}=\left(\mathrm{w}_{1}, \mathrm{w}_{2}, \ldots, \mathrm{w}_{\mathrm{n}}\right)$ is calculated by the HMM and the probability $P_{\text {grammar }}(\mathrm{W})$ of the word sequence $\mathrm{W}$ is calculated by the language model. This allows to calculate the probability of a word based on its context.

\subsection{Hamdani Mahdi [17]}

In this work the improvement of context dependent modeling for Arabic handwriting recognition is proposed. Since the number of parameters in context dependent models is huge, CART trees are used for state tying. This work is based on a new set of questions for the CART tree construction based on a "lossy mapping" categorization of the Arabic shapes. The used system is a combination of Hidden Markov Models and Recurrent Neural Networks using the hybrid approach.

The advantage of context dependent modeling is very clear; the writing style of current stroke is always affected by its context (previous and future strokes) figure 6.
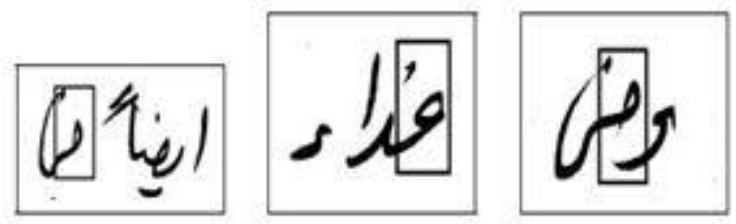

Figure 6: Example of context dependency in Arabic handwriting: the character ' $A$ in its beginning form is written differently in different contexts by the same writer.[17]

Modeling characters within context cause practical problems for parameter estimation. The number of free parameters is huge if compared to the case of context independent modeling. State tying is essential to reduce the number of parameters. State tying aims to determine the states which share the same Gaussian distributions in the context of GHMMs.

There are multiple methods for state tying. Decision trees are one of the most used methods in speech recognition systems; the objective is to tie the states which are similar. Decision trees are binary trees in which the inner nodes are tagged with questions and the leaves are tagged with class labels.

The questions used in the CART construction concern the data to be classified using the tree. The questions are generally predefined using prior knowledge about the data. There is no standard set of questions used for CART trees 
construction in Arabic handwriting recognition. The adopted list of question used in previous work "The RWTH large vocabulary Arabic handwriting recognition system 2014" [18] was based in a rough classification of the Arabic characters.

Figure 7 presents an example of the used CART tree:

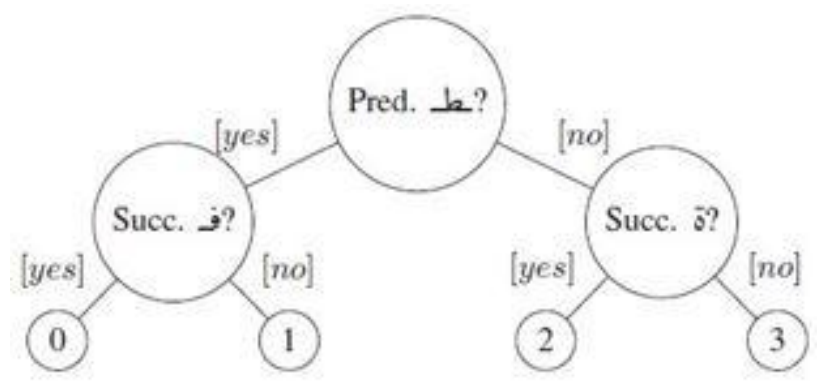

Figure 7: Example of a CART tree: the root of the tree contains the question "is the predecessor character belongs to the class $\frac{b}{2}$ ?", if the response is "yes" the next question that comes is "is the successor character belongs to the class -2 ?", if "yes" then the selected mixture index is " 0 " [17].

\subsection{Irfan Ahmad[19]}

Irfan presents an improvement in sub-character HMM modeling for Arabic text recognition, that allow sharing of common patterns between different position-dependent shape forms of an Arabic character as well as between different characters. The number of HMMs gets reduced considerably while still capturing the variations in shape patterns. This results in a compact, efficient, and robust recognizer with reduced model set. Additionally contextual sub-characters HMMs for text recognition and multi-stream contextual subcharacter HMMs are investigated where the features calculated from a sliding window frame form one stream and its derivative features are part of the second stream.

Sub character modeling: A character is split into subcharacters exploiting the similar patterns between different characters and their different position-dependent shapes. The sub-character patterns can then be used to reconstruct the character. This lead to huge reduction in number of basic HMMs which is important for many reasons like robust training with limited data, and a more compact recognizer.

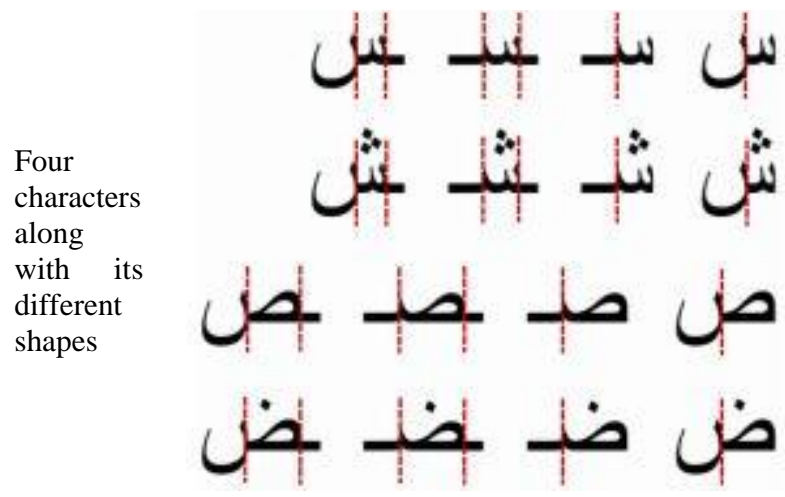

Five subcharacter patterns

(along

with

connector

and space

models)

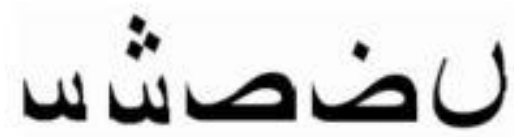

which can

represent

the above

four

characters

and their

different

shapes.

Figure 8: An example of sub-character modeling reducing the number of basic HMM models [19]

Contextual sub-character HMMs: The motivation for using contextual sub-character models also increases when looking at the nature of the Arabic script where the same strokes may be written differently depending on adjacent strokes.

The data driven approach is investigated to contextual subcharacter HMMs for Arabic text recognition. First, all the different contextual forms are generated from the noncontextual forms using the training transcription. Some contextual forms occur very few times in the training data and as such robustly estimating their parameters is difficult. Due to this reason, adequate level of tying is performed such that the tied parameters share the same pool of data during training.

- First step: tie the transition probabilities of all the contextual forms of a sub-character.

- Second: perform the training for the contextual forms using few iterations of the Baum-Welch training algorithm.

- Third : perform state tying between different contextual forms of the sub-character preserving the state sequence

State tying is an important step, especially when using continuous HMMs, so that the parameters get trained robustly with availability of few training samples.

\section{COMPARATIVE RESULTS}

The table below presents the comparative results of contextual handwritten recognition systems based HMMs for cursive Arabic and Latin script according to reference's system, to mention the impact of contextual approach in handwritten recognition field:

Table: Comparative results of contextual handwritten recognition systems

\begin{tabular}{llll}
\hline \multirow{2}{*}{ System } & \multirow{2}{*}{ Database/script } & \multicolumn{2}{c}{ Accuracy (\%) } \\
\cline { 3 - 4 } Fink [10] & IAM/Latin & Ref* & Ctxt* \\
EI-Hajj [12] & IFN-ENIT/Arabic & $\mathbf{7 6 . 0 0}$ & $\mathbf{7 7 . 5 0}$ \\
Bianne [15] & Rimes/Latin & $\mathbf{6 7 . 5 0}$ & $\mathbf{9 2 . 9 1}$ \\
Morillot [16] & Rimes/Latin & $\mathbf{6 8 . 8 0}$ & $\mathbf{7 3 . 8 0}$ \\
Hamdani [17] & OpenHart/Arabic & $\mathbf{7 7 . 2 0}$ & $\mathbf{8 0 . 1 0}$ \\
Irfane [19] & IFN-ENIT/Arabic & $\mathbf{9 5 . 3 8}$ & $\mathbf{9 6 . 6 7}$ \\
\hline
\end{tabular}

Ref: Reference's system; Ctxt: Contextual system 
From the table, it can be seen that there are significant improvement in the recognition results as compared to the reference's systems for all the proposed works cited in our study.

In ordre to investigate the potential of using context dependent sub words units for unconstrained offline handwritten recognition Fink [10] conducted a serie of experiments on a challenging task defined on the IAM database. As can be seen from the table, the performance could be increased significantly beyond the baseline. El-Hajj [12] evaluated the performance of his recognition system on the benchmark database IFN-ENIT. Results in table shown an improvement due to the contextual character modeling: accuracy is increased by $0.6 \%$ in absolute value which corresponds to a $7.8 \%$ reduction in error rate; this shows the effectiveness of modeling overlapped characters by specific models while keeping the total number of models manageable. The proposed work by Hamdani [17] had evaluated in OpenHart database [20]; an absolute improvement of $2.9 \%$ in terms of WER (Word Error Rate) is performed by using the context dependent labels and the lossy mapping CART. Irfane [19] proposed sub-character HMM models for Arabic text recognition that allow sharing of common patterns between different position-dependent shape forms of an Arabic character as well as between different character. The number of HMMs gets reduced considerably from 178 unique character-shapes to $97 \mathrm{HMMs}$ to model all the characters and their shape variations. This by itself is a great improvement in terms of system compactness and efficiency. The results show that there are significant improvements in the recognition results as compared to the baseline systems.

Bianne [15] presents an HMM-based recognizer for the offline recognition of handwritten words using contextual characters models: trigraphs. The system is shown to perform better than a baseline context independent system, and reaches an accuracy higher than $74 \%$ on the public available Rimes database [21][22]. The last work proposed by Morillot [16] describes a global approach for the recognition of handwritten lines; the main contribution is to extend the context-dependent HMMs word recognition system to the line level without segmentation into words and the obtained results are clearly significant.

\section{CONCLUSION AND PERSPECTIVES}

As the success of context dependent models in the domain of speech recognition was so triumphant there were no obvious reasons to doubt that this modeling technique would deliver vast performance improvements also in the related area of handwriting recognition. The advantage of context in handwriting recognition is very clear; the writing style of current stroke is always affected by its context (previous and next strokes).

In this paper we present a survey of offline handwriting recognition systems using context dependent modeling for cursive Arabic and Latin script, there are many approach using various techniques exploiting the context. Though significant improvement in performance could be achieved, the results show that context dependent modeling ameliorate the system performance. Better results could be obtained with the contextual sub character HMM system proposed by [Irfane14] that allows the sharing of common patterns between different shape forms of character as well as between different characters.
Modeling characters within context cause practical problems for parameter estimation. The number of free parameters is huge if compared to the case of context independent modeling, each character can have a multitude of different contexts, and the number of training data becomes too low. There are multiple methods to fix this problem based on clustering and sharing parameters between states.

Consequently, future research works should exploit this survey and benefit a priori of advantages of each approach while avoiding main disadvantages to make improvements and develop a system that meet expectations.

\section{REFERENCES}

[1] Réjean Plamondon, Sargur Srihari "On-line and off-line handwriting recognition: a comprehensive survey". in IEEE Transactions on Pattern Analysis and Machine Intelligence 22(1):63-84 · January 2000

[2] K. Gaurav and Bhatia P. K., "Analytical Review of Preprocessing Techniques for Offline Handwritten Character Recognition", $2^{\text {nd }}$ International Conference on Emerging Trends in Engineering \& Management, ICETEM, 2013

[3] Namrata Dave "Segmentation Methods for Hand Written Character Recognition" International Journal of Signal Processing, Image Processing and Pattern Recognition Vol. 8, No. 4 (2015), pp. 155-164

[4] B. El Qacimy A. Hammouch ; M. A. Kerroum "A review of feature extraction techniques for handwritten Arabic text recognition". Electrical and Information Technologies (ICEIT), 2015 International Conference

[5] Mohamed Abaynarh and Lahbib Zenkouar, "Offline Handwritten Characters Recognition Using Moments Features and Neural Networks". Computer Technology and Application 6 (2015)

[6] Priya Sharma, Randhir Singh "Survey and Classification of Character Recognition System" International Journal of Engineering Trends and Technology- Volume4Issue3- 2013

[7] Bhatia Neetu, "Optical Character Recognition Techniques",International Journal of Advanced Research in Computer Science and Software Engineering, Volume 4, Issue 5, May 2014.

[8] A.Lawgali : "A Survey on Arabic Character Recognition" International Journal of Signal Processing, Image Processing and Pattern Recognition. Vol. 8, No. 2 (2015), pp. 401-426.

[9] Youssouf Chherawala, Partha Pratim Roy, Mohamed Cheriet "Context-dependent BLSTM models. Applications to offline handwriting recognition" 2014 IEEE International Conference on Image Processing (ICIP)

[10] Gernot A. Fink and Thomas Plotz, "On the use of context-dependent modeling units for HMM-based offline handwriting recognition," in Proceedings of the 9th International Conference on Document Analysis and Recognition, 2007, vol. 2 of ICDAR '07, pp. 729-733.

[11] U.-V. Marti and H. Bunke. The IAM-database: An English sentence database for offline handwriting recognition. Int.Journal on Document Analysis and Recognition, 5(1):39- 46, 2002. 
[12] Ramy El-Hajj, Chafic Mokbel, and Laurence Likforman-Sulem, "Recognition of Arabic handwritten words using contextual character models," in DRR, Berrin A. Yanikoglu and Kathrin Berkner, Eds. 2009, vol. 6815 of SPIE Proceedings, p. 681503, SPIE.

[13] M. Pechwitz, S. S. Maddouri, V. Märgner, N. Ellouze, and H. Amiri, "IFN/ENIT - Database of Handwritten Arabic Words," in 7th Colloque International Francophone sur l'Ecrit et le Document, CIFED 2002, 2002, pp. 129-136.

[14] EL-Hajj R, Mokbel.C Likforman-Sulem L “ Arabic handwriting recognition using baselin dependent features and hidden Markov modeling" Proceedings of the Eighth International Conference on Document Analysis and Recognition ICDAR05, p 893-897, 2005.

[15] Anne-Laure Bianne-Bernard, Far`es Menasri, Ramy AlHajj Mohamad, Chafic Mokbel, Christopher Kermorvant, and Laurence Likforman-Sulem, "Dynamic and contextual information in HMM modeling for handwritten word recognition," IEEE Transactions on Pattern Analysis and Machine Intelligence, vol. 33, no. 10, pp. 2066-2080, 2011.

[16] Olivier Morillot, Emmanuèle Grosicki , Laurence Likforman-Sulem, «Reconnaissance de courriers manuscrits par HMM contextuels et modèle de langage » DOI:10.3166/DN.16.2.69-90 (C) 2013 Lavoisier Document numérique - no 2/2013, 69-90
[17] Mahdi Hamdani, Patrick Doetsch and Hermann Ney "Improvement of Context Dependent Modeling for Arabic Handwriting Recognition “ $201414^{\text {th }}$ International Conference on frontiers in Handwrting Recognition.

[18] M. Hamdani, P. Doetsch, M. Kozielski, A. El-Desoky Mousa, and H. Ney, "The RWTH large vocabulary Arabic handwriting recognition system," in International Workshop on Document Analysis Systems, Tours `a Loire Valley, France, Apr. 2014.

[19] Irfan Ahùad, Gernot A.Fink and Sabri A.Mahmoud "Improvement in Sub-character HMM Model Based Arabic Text Recognition" $201414^{\text {th }}$ International Conference on frontiers in Handwrting Recognition.

[20] A. Tong, M. Przybocki, V. Maergner, and H. El Abed, "NIST 2013 Open Handwriting Recognition and Translation evaluation", Proceedings of the NIST 2013 Open Handwriting and Recognition Workshop, 2013, in press.

[21] E. Augustin, M. Carre, E. Grosicki, J.-M. Brodin, E. Geoffrois, and F. Preteux, "Rimes Evaluation Campaign for Handwritten Mail Processing," Proc. Int'l Workshop Frontiers in Handwriting Recognition, pp. 231-235, 2006.

[22] http://rimes.it sudparis.eu/, 2010. 